\title{
OPTIMAL DECISION IN MC SUPPLY CHAIN WITH OVERCONFIDENT RETAILER BASED ON THE NEWSVENDOR MODEL
}

\author{
Bayi Cheng ${ }^{1,2, *}$, Ruofan Li $^{1,2}$, Xiaoxi Zhu ${ }^{1,2}$, Mi Zhou ${ }^{1,2}$ and Xiongfei CaO ${ }^{1,2}$
}

\begin{abstract}
In this paper, we analyze the optimal order-quantity decisions in a supply chain with mass customization (MC) manufacturer and overconfident retailers. First, we consider a newsvendor model in which an unbiased retailer sells mass customized products. The retailer needs to make order quantity decisions before the selling season. Meanwhile, the supplier is a mass customization manufacturer and implements modular production. The supply process is uncertain, as the real quantity the retailer received is the order quantity multiplied by a random yield rate. Second, two overconfident models are considered and theorems are proposed. In the first model, the behavioral bias of overconfidence only affects the retailer's judgment of variance of market demand. In the second model, the behavior bias of overconfidence affects not only the retailer's cognition of the variance of market demand, but also his cognition of the expectation of market demand. In addition, the relationship between the optimal decisions and the modularity level is obtained. Finally, we provide managerial insights for the decision makers of the retailers and the manufacturers on order quantity and modularity level, respectively.
\end{abstract}

Mathematics Subject Classification. 90B05, 97M40.

Received May 14, 2020. Accepted April 7, 2021.

\section{INTRODUCTION}

With the substantial improvement of living standards and the rapid development of economy, the consumers are no longer satisfied with unchanging and low-cost products. They start to pursue diversified and personalized products. Mass customization helps enterprises to cope with the increasingly uncertain, competitive and complex market environment, and keep their business in line with customer needs. Accordingly, product customization is an inevitable trend of future business development. In the context of mass customization, we study the supply chain decision making and consider one of the behavioral biases-overconfidence. Overconfidence theory is a behavioral finance theory proposed at the end of the 20th century. Overconfidence is a universal psychological phenomenon. The term of "overconfidence" comes from research in cognitive psychology. A large amount of literature in cognitive psychology holds that people are overconfident, especially in the accuracy of their own knowledge. People systematically underestimate certain types of information and overestimate others.

\footnotetext{
Keywords. Overconfidence, mass customization, newsvendor model, decision analysis.

1 School of Management, Hefei University of Technology, Hefei 230009, P.R. China.

2 Key Laboratory of Process Optimization and Intelligent Decision-making, Ministry of Education, Hefei 230009, P.R. China.

* Corresponding author: chengbayi@hotmail.com
} 
The supply chain with mass customization and the optimal decisions with overconfidence retailer based on newsvendor model are both interesting and worth studying. The scholars do numerous studies and meaningful research on them.

Some scholars put forward their views on the concept and status of mass customization. Mass customization has become an important manufacturing strategy. And the $\mathrm{MC}$ concept, which focuses on changing requirements and technologies, is increasingly important. Mass customization is also one of the main strategies adopted in today's highly competitive manufacturing industry. The concept of mass customization has received considerable attention in the research literature in recent years $[6,17,26,28]$. How to deal with the contradiction between the effect of mass production and the demand for customization is a key issue in the study of mass customization [31]. MacCarthy et al. [17] develop a taxonomy of operational modes for mass customization and identify five fundamental modes of operation for mass customization.

There are also numerous studies on the supply chain with mass customization. Yao and Liu [31] summarize the dominant contradictions of the supply chain scheduling in mass customization and the ways to relieve them. $\mathrm{Xu}$ et al. [28] propose a decision support model to obtain the optimal production plan in mass customization. Jost and Ssser [12] find that the manufacturer finds providing customization is always optimal, and show how the optimal degree of customization is affected by the interaction of market and production factors. In the above supply chain research, there are some specific studies on the pricing and value of mass customization. Alptekinoglu and Corbett [1] find that a mass producer facing competition from a mass customizer provides lower product varieties than a mass producer monopolist to reduce the intensity of price competition. Choi et al. [4] investigate the optimal pricing problem in a mass customization supply chain with one risk averse manufacturer and two risk averse competing retailers. Fei and Zhao [5] study behavioral pricing in a competitive environment, which includes a standard product firm and a custom product firm. Merle et al. [18] argue that providing efficient customization is not sufficient in itself to assess the value of mass customization. And they focus on the benefits perceived by individual consumers.

A large part of the research on mass customization is on the level of mass customization. In a game-theoretic model of mass customization, the degree of company-customer interaction is defined as level of mass customization [12]. In this paper, we define the level of modularity as level of mass customization. The concept of modularity has been widely accepted because of mass customization and the need for shorter development time in the manufacturing process [2]. Zhang et al. [34] propose a model to study the impact of mass customization and product modularization on supply chain quality integration. Bi and Zhang [2] introduce the classification of modular applications, research problems and design methods.

Human beings are critical to the functioning of most operating systems. Because the people influence how these systems work and how they are executed. However, the people who participate in operating systems are not completely rational [7]. People have cognitive biases. As one of the most consistent, powerful, and widespread cognitive biases, Li et al. [16] overconfidence has attracted extensive attention and research in the field of economics [8] and operations management [23]. Decision makers with overconfidence perceive the expected outcome of an uncertain event is more certain than it might be [15]. We focus on the study of overconfidence in behavioral operations management and supply chains. Xu et al. [29] find that the higher the overconfident level is, the higher selling price that the overconfident retailer charges in a duopolistic market with uncertain demand. Jain et al. [11] study the impact of this overconfidence bias on the buyer's supply-based design strategy. Jiang and Liu [10] consider that management optimism has become ubiquitous in a fiercely competitive market and a company should hire a realistic manager. Li [15] studies the effects associated with overconfidence in distribution channels. He finds overconfidence bias leads to a lower expected profit for a centralized channel but enhances the performance of a decentralized channel.

On the other hand, the newsvendor problem has a rich history, dating back to the 19th century. However, it didn't become a topic of serious and extensive study by academicians until the 1950s [21]. Yao et al. [32] show how the newsvendor problem with joint pricing and order decision can be solved without using specific demand functions. Kocabiyikoglu et al. [14] extend the traditional framework of the price-setting newsvendor problem. They allow the decision maker to determine both the selling price and the order quantity. Ovchinnikov 
et al. [20] extend the standard theory of newsvendor competition by including an optimal best-response policy for competing with a behaviorally biased newsvendor. Xue et al. [30] consider the diversification strategy for a mean variance risk-sensitive manufacturer with unreliable suppliers based on the linear model and newsvendor model, respectively.

In the emerging field of behavior operation, a part of the literature studies take the newsvendor model as the research subject, mainly analyzing the influence of behavior on the decision-making in the newsvendor model. Moore and Healy [19] define three distinct ways of overconfidence: over-estimation of one's actual performance, over-placement of one's performance relative to others, and over-precision in one's beliefs. An overconfident newsvendor model is developed by defining the overconfidence as the newsvendor's over-precision about the true demand [33]. Croson et al. [3] show that overconfident newsvendors place suboptimal orders and earn lower profits than well-calibrated newsvendors. Ren and Croson [23] provide two experiments supporting the theoretical conclusion, that is, underestimating the variance of demand causes orders to deviate from optimal. Ren et al. [24] further demonstrate that order bias is linear in the level of overconfidence, and is increasing with the variance of the demand distribution. Li et al. [16] study the effects and implications of overconfidence in a competitive newsvendor setting. They find that when the product s profit margin is high, overconfidence can lead to a first-best outcome. Kirshner and Shao [13] show how probability weighting functions can be used to jointly model optimism and overconfidence in the newsvendor problem.

In this paper, we analyze the optimal order-quantity decisions in a mass customization supply chain with mass customization manufacturer and overconfident retailers. Our contribution is considering the behavioral bias of overconfidence in the decision-making study of the mass customization supply chain. At the same time, we take into account both the level of mass customization and supply uncertainty, that is, the reliable level of the manufacturer.

\section{BASIC MODEL DESCRIPTION}

In this section, we consider a two-stage supply chain based on newsvendor model. The supply chain includes an MC manufacturer and a downstream retailer. The retailer sells the MC product to the final consumers to satisfy the stochastic demand $X$. The manufacturer is unreliable in the sense that the manufacturer's delivered quantity may be strictly lower than the quantity ordered by the retailer. In line with the classic newsvendor model, we assume that the retailer's order quantity decision should be made before the random demand is realized. The retailer needs to decide the order quantity to maximize his profit.

In this paper, the manufacturer is a monopoly manufacturer of an MC product. And modular production is applied to achieve mass customization for the manufacturer. Let $m$ denote the modularity level of the manufacturer, which represents the mass customization capability of the manufacturer and $m>0$. We assume that wholesale price $w$ has a positive linear correlation with modularity level $m$. Let $\varepsilon$ be the modularity level-wholesale price sensitive coefficient, and $\varphi$ be the wholesale price independent of modularity level. So, the wholesale price the retailer receives from the manufacturer is $w=\varphi+\varepsilon m$. Then we use the proportional yield model to measure the manufacturer's unreliable risk. Specifically, let $q$ be the order quantity from the retailer, and $R \in[0,1]$ be the yield rate of the MC manufacturer, that is, the MC manufacturer's reliable level. Then, the quantity the retailer receives from the manufacturer is $R q$. We assume the stochastic demand $X$ has a cumulative density function $(\mathrm{CDF}) F_{X}(x)$ and a probability density function (PDF) $f(x)$. Denote $\mu=E[X]$, $\sigma=\operatorname{Var}[X]$ (Fig. 1).

Let $p$ be the unit selling price of the MC product, and $v$ be the unit reduced price. Without loss of generality, we assume that $p \geq w \geq v$. The holding cost or salvage cost of the retailer given order quantity $q$ is $v(R q-X)^{+}$, where $(\cdot)^{+}=\max (\cdot, 0)$. We assume that retailer's revenue is $p \min \{X, R q\}$, which depends on the order quantity the retailer receives from the manufacturer. Then, the profit function with uncertain demand for the retailer, $\Pi$, is given by

$$
\begin{aligned}
\Pi & =p \min \{X, R q\}-(\varphi+\varepsilon m) R q+v(R q-X)^{+} \\
& =[p-(\varphi+\varepsilon m)] R q-(p-v)(R q-X)^{+} .
\end{aligned}
$$




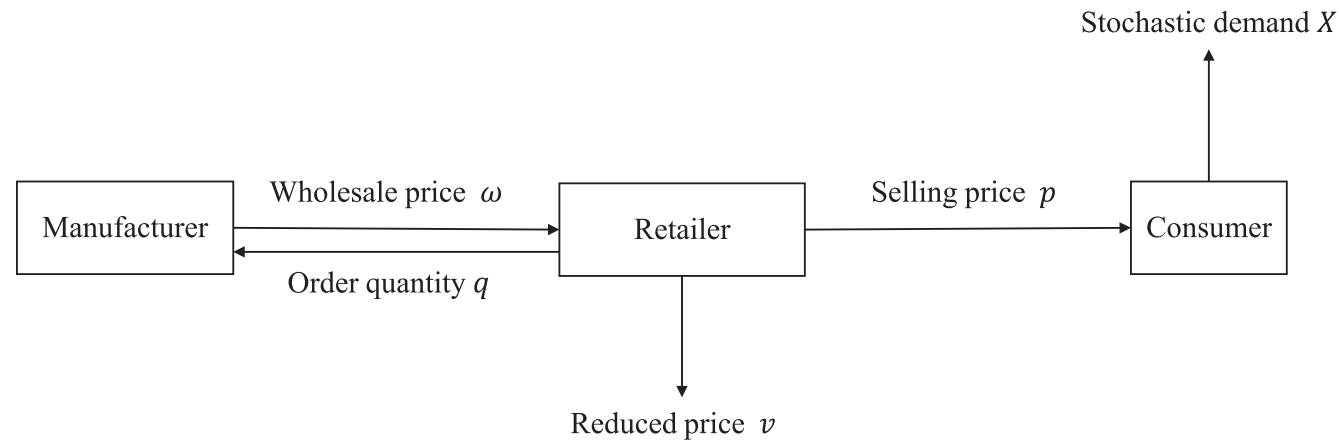

FiguRE 1. Basic supply chain.

In Sections 3 and 4, we discuss the case of overconfidence retailer. Denote $\alpha$ be the measure of overconfidence. The overconfident retailer is a retailer that exhibits cognitive bias by making order decision as though the demand were $D$ rather than $X$, where

$$
D=\alpha \mu+(1-\alpha) X
$$

for $\alpha \in[0,1]$. We assume $D$ has a cumulative density function $(\mathrm{CDF}) F_{D}(x)$. If $\alpha>0$, the retailer is overconfident and considers that demand is less variable than $X$. In the extreme, $\alpha=1$ means that the retailer regard demand as constant and equal to its mean. At the other extreme, $\alpha=0$ indicates the retailer is unbiased.

We first study the optimal order decision of unbiased retailer in MC-supply chain. Based on the above discussion and assumptions, we have the retailer's expected profit

$$
E[\Pi]=\int_{0}^{R q}\{[p-(\varphi+\varepsilon m)] x-[(\varphi+\varepsilon m)-v](R q-x)\} f(x) \mathrm{d} x+\int_{R q}^{+\infty}[p-(\varphi+\varepsilon m)] R q f(x) \mathrm{d} x .
$$

The optimal order quantity shall satisfy:

$$
\begin{aligned}
\frac{\mathrm{d} E[\Pi]}{\mathrm{d} q} & =-R[(\varphi+\varepsilon m)-v] \int_{0}^{R q} f(x) \mathrm{d} x+R[p-(\varphi+\varepsilon m)] \int_{R q}^{+\infty} f(x) \mathrm{d} x \\
& =0 .
\end{aligned}
$$

Then we have

$$
\frac{\int_{0}^{R q} f(x) \mathrm{d} x}{\int_{R q}^{+\infty} f(x) \mathrm{d} x}=\frac{p-(\varphi+\varepsilon m)}{(\varphi+\varepsilon m)-v} .
$$

That is,

$$
\int_{0}^{R q} f(x) \mathrm{d} x=\frac{p-(\varphi+\varepsilon m)}{p-v} .
$$

Obviously we have the following lemma.

Lemma 2.1. Given the MC manufacturer's reliable level $R$,

(a) The unbiased retailer's optimal order quantity $q^{*}=R^{-1} F_{X}^{-1}(\beta)$, where $\beta=\frac{p-(\varphi+\varepsilon m)}{p-v}$.

(b) The unbiased retailer's optimal order quantity $q^{*}$ is decreasing in the modularity level $m$.

Proof. (a) From the above analysis, we know that the unbiased retailer's optimal order quantity $q^{*}$ meet $\int_{0}^{R q^{*}} f(x) \mathrm{d} x=\frac{p-(\varphi+\varepsilon m)}{p-v}$. According to the relationship between the distribution function and the probability density function, the above expression can be converted into: $F_{X}\left(R q^{*}\right)=\frac{p-(\varphi+\varepsilon m)}{p-v}$. We can get $R q^{*}=F_{X}^{-1}\left(\frac{p-(\varphi+\varepsilon m)}{p-v}\right)$ by the definition of inverse function. That is, $q^{*}=R^{-1} F_{X}^{-1}\left(\frac{p-(\varphi+\varepsilon m)}{p-v}\right)$ is proved. 
(b) From the proof of Lemma 2.1a, we know that $F_{X}\left(R q^{*}\right)=\frac{p-(\varphi+\varepsilon m)}{p-v}$. Then we can learn that the manufacturer's modularity level $m$ increases, the value of $F_{X}\left(R q^{*}\right)$ decreases. Because of The monotony undiminished property of the distribution function, the value of $R q^{*}$ decreases. Therefore, the unbiased retailer's optimal order quantity $q^{*}$ is decreasing in the modularity level $m$.

Lemma 2.2. Given the $M C$ manufacturer's reliable level $R$, the unbiased retailer's optimal expected profit $\Pi^{*}=[p-(\varphi+\varepsilon m)] R q^{*}-(p-v) \int_{0}^{R q^{*}} F_{X}(x) \mathrm{d} x$.

Proof. From (2.1), the expected profit function for the retailer can be transformed into

$$
E[\Pi]=[p-(\varphi+\varepsilon m)] R q-(p-v) E\left[(R q-X)^{+}\right]
$$

where $E\left[(R q-X)^{+}\right]=\int_{0}^{R q}(R q-x) \mathrm{d} F_{X}(x)=\int_{0}^{R q} F_{X}(x) \mathrm{d} x$. Then, we obtain the expected profit for the retailer

$$
E[\Pi]=[p-(\varphi+\varepsilon m)] R q-(p-v) \int_{0}^{R q} F_{X}(x) \mathrm{d} x .
$$

We learn that the unbiased retailer's optimal order quantity $q^{*}$ from Lemma 2.1. Thus, the optimal expected profit

$$
\Pi^{*}=[p-(\varphi+\varepsilon m)] R q^{*}-(p-v) \int_{0}^{R q^{*}} F_{X}(x) \mathrm{d} x .
$$

\section{BASIC OVERCONFIDENCE MODEL}

After reviewing much of the literature on overconfidence, Moore and Healy [19] define three distinct ways of overconfidence: over-estimation, over-placement, and over-precision. The latter type means that overconfident people overestimate how accurate their predictions are. That is, they believe they are better at judging things than they really are.

Based on this, we assume an overconfident retailer whose perception of market demand information is overly accurate in MC-supply chain. The overconfident retailer believes the variance of random demand fluctuations is smaller than the actual value. They behave as though demand is less variable than $X[16]$. According to the assumptions in Section 2, the overconfident retailer's belief in market demand is $D=\alpha \mu+(1-\alpha) X$ for $\alpha \in[0,1]$. We easily obtained $E[D]=\mu, \operatorname{Var}[D]=(1-\alpha)^{2} \sigma^{2}$. That is, the overconfident retailer's judgment of the mean value of random market demand is correct, but his judgment of the variance of random market demand is less than the actual value. The parameter $\alpha$ is the level of overconfidence, reflecting the degree of deviation of retailer's cognition of demand information (Fig. 2).

Then, the faith profit function for the overconfident retailer, $\hat{\Pi}_{\alpha}$, is given by

$$
\begin{aligned}
\hat{\Pi}_{\alpha} & =p \min \left\{D, R q_{\alpha}\right\}-(\varphi+\varepsilon m) R q_{\alpha}+v\left(R q_{\alpha}-D\right)^{+} \\
& =[p-(\varphi+\varepsilon m)] R q_{\alpha}-(p-v)\left(R q_{\alpha}-D\right)^{+} .
\end{aligned}
$$

From Lemma 2.2, we obtain the overconfident retailer's belief expected profit

$$
E\left[\hat{\Pi}_{\alpha}\right]=[p-(\varphi+\varepsilon m)] R q_{\alpha}-(p-v) \int_{0}^{R q_{\alpha}} F_{D}(x) \mathrm{d} x
$$

and the overconfident retailer's resulting expected profit

$$
E\left[\Pi_{\alpha}\right]=[p-(\varphi+\varepsilon m)] R q_{\alpha}-(p-v) \int_{0}^{R q_{\alpha}} F_{X}(x) \mathrm{d} x .
$$




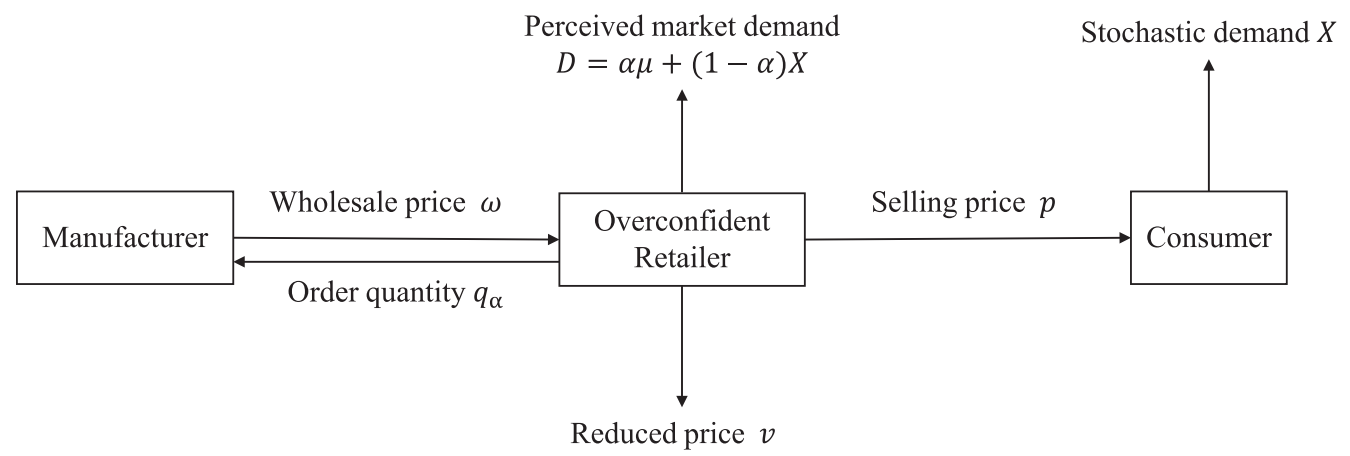

FigURE 2. Basic overconfidence supply chain.

The overconfident retailer's optimal resulting expected profit $\Pi_{\alpha}^{*}$ when choosing optimal order quantity $q_{\alpha}^{*}$ is

$$
\Pi_{\alpha}^{*}=[p-(\varphi+\varepsilon m)] R q_{\alpha}^{*}-(p-v) \int_{0}^{R q_{\alpha}^{*}} F_{X}(x) \mathrm{d} x .
$$

The relationship between the overconfident retailer's optimal order quantity $q_{\alpha}^{*}$ and the unbiased retailer's optimal order quantity $q^{*}$ is given by the following Theorem 3.1 .

Theorem 3.1. Given the MC manufacturer's reliable level $R$,

(a) The overconfident retailer's optimal order quantity $q_{\alpha}^{*}=R^{-1} \alpha \mu+(1-\alpha) q^{*}$.

(b) The overconfident retailer's optimal order quantity $q_{\alpha}^{*}$ is decreasing in the modularity level $m$.

Proof. (a) From Lemma 2.1, the unbiased retailer's optimal order quantity $q^{*}=R^{-1} F_{X}^{-1}(\beta)$. That is, $F_{X}\left(R q^{*}\right)=\beta$, where $\beta$ is the critical fractile. The overconfident retailer with overconfidence level $\alpha$ orders $q_{\alpha}^{*}$ such that $F_{D}\left(R q_{\alpha}^{*}\right)=\beta$ when the demand is $X$. Then, we have

$$
F_{D}\left(R q_{\alpha}^{*}\right)=P\left(\alpha \mu+(1-\alpha) X \leq R q_{\alpha}^{*}\right)=F_{X}\left(\frac{R q_{\alpha}^{*}-\alpha \mu}{1-\alpha}\right) .
$$

We obtain $R q^{*}=\frac{R q_{\alpha}^{*}-\alpha \mu}{1-\alpha}$. We simplify to get

$$
q_{\alpha}^{*}=R^{-1} \alpha \mu+(1-\alpha) q^{*} .
$$

(b) Moreover, $\beta=\frac{p-(\varphi+\varepsilon m)}{p-v}$, then we can learn that the overconfident retailer's optimal order quantity $q_{\alpha}^{*}$ is decreasing in the modularity level $m$.

Theorem 3.1a indicates that the overconfident retailer's optimal order quantity $q_{\alpha}^{*}$ is a linear function of the unbiased retailer's optimal order quantity $q^{*}$. Corollary 3.2 illustrates the variation rule of the overconfident retailer's optimal order quantity $q_{\alpha}^{*}$ with its overconfident level $\alpha$, as well as the relationship between the overconfident retailer's optimal order quantity $q_{\alpha}^{*}$ and the unbiased retailer's optimal order quantity $q^{*}$. In Corollary 3.2, we quote the definition of the market environment from Schweitzer and Cachon [25].

Corollary 3.2. Given the MC manufacturer's reliable level $R$,

(a) Under a high-profit condition $\left(q^{*}>\mu\right), \frac{\partial q_{\alpha}^{*}}{\partial \alpha}<0$ and $q_{\alpha}^{*}<q^{*}$ when $q^{*}>R^{-1} \mu ; \frac{\partial q_{\alpha}^{*}}{\partial \alpha}>0$ and $q_{\alpha}^{*}>q^{*}$ when $\mu<q^{*}<R^{-1} \mu$. 
(b) Under a low-profit condition $\left(q^{*}<\mu\right), \frac{\partial q_{\alpha}^{*}}{\partial \alpha}>0$ and $q_{\alpha}^{*}>q^{*}$ in all cases.

Proof. From Theorem 3.1a, the overconfident retailer's optimal order quantity $q_{\alpha}^{*}=R^{-1} \alpha \mu+(1-\alpha) q^{*}$. Then, we obtain

$$
\begin{aligned}
\frac{\partial q_{\alpha}^{*}}{\partial \alpha} & =R^{-1} \mu-q^{*} \\
q_{\alpha}^{*}-q^{*} & =\alpha\left(R^{-1} \mu-q^{*}\right) .
\end{aligned}
$$

Thus, when $q^{*}>R^{-1} \mu, \frac{\partial q_{\alpha}^{*}}{\partial \alpha}<0$ and $q_{\alpha}^{*}<q^{*}$; when $\mu<q^{*}<R^{-1} \mu, \frac{\partial q_{\alpha}^{*}}{\partial \alpha}>0$ and $q_{\alpha}^{*}>q^{*}$. Further, based on Schweitzer and Cachon's definition of the market environment, Corollary 3.2 can be obtained.

Corollary 3.2 indicates that in MC-supply chain the overconfident retailer's optimal order quantity is decreasing in its overconfidence level when the unbiased retailer's optimal order quantity is greater than the mean of random demand (the retailer is in a high-profit condition). Meanwhile, the overconfident retailer tends to place fewer orders than the unbiased retailer. The overconfident retailer's optimal order quantity is increasing in its overconfidence level in two cases. One case is when the unbiased retailer's optimal order quantity is greater than the mean of random demand and less than the ratio of the mean of random demand to the MC manufacturer's reliable level. The other case is when the unbiased retailer's optimal order quantity is less than the mean of random demand (the retailer is in a low-profit condition). Meanwhile, the overconfident retailer tends to place more orders than the unbiased retailer. The deviation value between the order quantity of the overconfident retailer and that of the unbiased retailer is increasing of the overconfident retailer's overconfidence level.

The above part is the analysis of the ordering decision of the overconfident retailer. Then, we try to learn about the expected profit of the overconfident retailer, including the resulting expected profit and the belief expected profit.

Theorem 3.3. Given the MC manufacturer's reliable level $R$,

(a) The overconfident retailer's optimal resulting expected profit $\Pi_{\alpha}^{*}$ is always less than the unbiased retailer's optimal expected profit $\Pi^{*}$; i.e., $\Pi_{\alpha}^{*} \leq \Pi^{*}$;

(b) The overconfident retailer's optimal resulting expected profit $\Pi_{\alpha}^{*}$ is decreasing and concave in $\alpha$; i.e., $\frac{\partial \Pi_{\alpha}^{*}}{\partial \alpha} \leq 0$ and $\frac{\partial^{2} \Pi_{\alpha}^{*}}{\partial \alpha^{2}} \leq 0$.

Proof. (a) From (2.8), we learn the expected profit for the retailer $E[\Pi]=[p-(\varphi+\varepsilon m)] R q-(p-$ v) $\int_{0}^{R q} F_{X}(x) \mathrm{d} x$. Then, we obtain

$$
\begin{aligned}
& \frac{\partial E[\Pi]}{\partial q}=[p-(\varphi+\varepsilon m)] R-(p-v) R F_{X}(R q) \\
& \frac{\partial^{2} E[\Pi]}{\partial q^{2}}=-(p-v) R^{2} f(R q) \leq 0 .
\end{aligned}
$$

Obviously, the retailer's expected profit function is a concave function of the order quantity $q$ and maximizes at the optimal order quantity $q^{*}$. Thus, whether the overconfident retailer's optimal order quantity $q_{\alpha}^{*}$ is higher or lower than the unbiased retailer's optimal order quantity $q^{*}$, it's always true that the overconfident retailer's resulting expected profit $\Pi_{\alpha}^{*}$ is less than the unbiased retailer's optimal expected profit $\Pi^{*}$.

(b) From (3.4) and (3.6), the overconfident retailer's optimal resulting expected profit

$$
\Pi_{\alpha}^{*}=[p-(\varphi+\varepsilon m)] R\left[R^{-1} \alpha \mu+(1-\alpha) q^{*}\right]-(p-v) \int_{0}^{R\left[R^{-1} \alpha \mu+(1-\alpha) q^{*}\right]} F_{X}(x) \mathrm{d} x .
$$


We take the derivative of $\alpha$.

$$
\begin{aligned}
\frac{\partial \Pi_{\alpha}^{*}}{\partial \alpha} & =[p-(\varphi+\varepsilon m)] R\left(R^{-1} \mu-q^{*}\right)-(p-v) R\left(R^{-1} \mu-q^{*}\right) F_{X}\left(R\left[R^{-1} \alpha \mu+(1-\alpha) q^{*}\right]\right) \\
& =(p-v) R\left(R^{-1} \mu-q^{*}\right)\left[\frac{p-(\varphi+\varepsilon m)}{p-v}-F_{X}\left[R\left(R^{-1} \alpha \mu+(1-\alpha) q^{*}\right)\right]\right] \\
& =(p-v) R\left(R^{-1} \mu-q^{*}\right)\left[F_{X}\left(R q^{*}\right)-F_{X}\left(R q_{\alpha}^{*}\right)\right] .
\end{aligned}
$$

By the proof of Corollary 3.2, we learn that $q_{\alpha}^{*}<q^{*}$ when $q^{*}>R^{-1} \mu, q_{\alpha}^{*}>q^{*}$ when $\mu<q^{*}<R^{-1} \mu$ and $q_{\alpha}^{*}>q^{*}$ when $q^{*}<\mu$. In addition, the stochastic demand's cumulative density function $F_{X}(x)$ is an increasing function. Thus, $\left(R^{-1} \mu-q^{*}\right)\left[F_{X}\left(R q^{*}\right)-F_{X}\left(R q_{\alpha}^{*}\right)\right] \leq 0$ in all cases. Then we can easily get $\frac{\partial \Pi_{\alpha}^{*}}{\partial \alpha} \leq 0$. Furthermore,

$$
\begin{aligned}
\frac{\partial^{2} \Pi_{\alpha}^{*}}{\partial \alpha^{2}} & =-(p-v) R\left(R^{-1} \mu-q^{*}\right)\left(\mu-R q^{*}\right) f\left[R\left(R^{-1} \alpha \mu+(1-\alpha) q^{*}\right)\right] \\
& =-(p-v)\left(\mu-R q^{*}\right)^{2} f\left(R q_{\alpha}^{*}\right) \leq 0 .
\end{aligned}
$$

Theorem 3.3 asserts that in MC-supply chain there is always a profit loss for the overconfident retailer, because the overconfident retailer's optimal order quantity always deviates from the unbiased retailer's optimal order quantity. Meanwhile, the overconfident retailer's optimal resulting expected profit decreases in its overconfidence level. And the higher the overconfidence level is, the faster the optimal profit decreases.

Corollary 3.4. Given the MC manufacturer's reliable level $R$,

(a) The overconfident retailer's optimal belief expected profit $\hat{\Pi}_{\alpha}^{*}$ satisfies $\hat{\Pi}_{\alpha}^{*}=(1-\alpha) \Pi^{*}+[p-(\varphi+\varepsilon m)] \alpha \mu$;

(b) The overconfident retailer's optimal belief expected profit $\hat{\Pi}_{\alpha}^{*}$ is increasing in $\alpha$; i.e., $\frac{\partial \hat{\Pi}_{\alpha}^{*}}{\partial \alpha} \geq 0$;

(c) The overconfident retailer's optimal belief expected profit $\hat{\Pi}_{\alpha}^{*}$ is always greater than the unbiased retailer's optimal expected profit $\Pi^{*}$; i.e., $\hat{\Pi}_{\alpha}^{*} \geq \Pi^{*}$. And $\hat{\Pi}_{\alpha}^{*}=\Pi^{*}$ if and only if $\alpha=0$.

Proof. (a) When the retailer is overconfident, his belief expected profit is calculated based on his belief in random demand $D$. The corresponding distribution function is $F_{D}(x)$. Thus, the overconfident retailer's optimal belief expected profit $\hat{\Pi}_{\alpha}^{*}=[p-(\varphi+\varepsilon m)] R q_{\alpha}^{*}-(p-v) \int_{0}^{R q_{\alpha}^{*}} F_{D}(x) \mathrm{d} x$. We take the integral transformation of $x=\alpha \mu+(1-\alpha) y$ and obtain

$$
\begin{aligned}
\hat{\Pi}_{\alpha}^{*} & =[p-(\varphi+\varepsilon m)] R q_{\alpha}^{*}-(p-v) \int_{0}^{R q_{\alpha}^{*}} F_{D}(x) \mathrm{d} x \\
& =[p-(\varphi+\varepsilon m)] R q_{\alpha}^{*}-(p-v) \int_{0}^{R q^{*}} F_{D}(\alpha \mu+(1-\alpha) y) \mathrm{d}(\alpha \mu+(1-\alpha) y) \\
& =[p-(\varphi+\varepsilon m)] R q_{\alpha}^{*}-(p-v)(1-\alpha) \int_{0}^{R q^{*}} F_{X}(y) \mathrm{d} y .
\end{aligned}
$$

From Theorem 3.1, we learn $q_{\alpha}^{*}=R^{-1} \alpha \mu+(1-\alpha) q^{*}$. Thus,

$$
\begin{aligned}
\hat{\Pi}_{\alpha}^{*} & =[p-(\varphi+\varepsilon m)] R\left[R^{-1} \alpha \mu+(1-\alpha) q^{*}\right]-(p-v)(1-\alpha) \int_{0}^{R q^{*}} F_{X}(y) \mathrm{d} y \\
& =(1-\alpha)\left[(p-(\varphi+\varepsilon m)) R q^{*}-(p-v) \int_{0}^{R q^{*}} F_{X}(y) \mathrm{d} y\right]+[p-(\varphi+\varepsilon m)] \alpha \mu \\
& =(1-\alpha) \Pi^{*}+[p-(\varphi+\varepsilon m)] \alpha \mu .
\end{aligned}
$$


(b) From the above proof, we take the derivative of $\alpha$ in $\hat{\Pi}_{\alpha}^{*}$ to obtain

$$
\begin{aligned}
\frac{\partial \hat{\Pi}_{\alpha}^{*}}{\partial \alpha} & =[p-(\varphi+\varepsilon m)] \mu-\Pi^{*} \\
& =[p-(\varphi+\varepsilon m)] \mu-\left[(p-(\varphi+\varepsilon m)) R q^{*}-(p-v) \int_{0}^{R q^{*}} F_{X}(x) \mathrm{d} x\right] \\
& =[(\varphi+\varepsilon m)-v] \int_{0}^{R q^{*}} F_{X}(x) \mathrm{d} x+[p-(\varphi+\varepsilon m)]\left[\mu-\left(R q^{*}-\int_{0}^{R q^{*}} F_{X}(x) \mathrm{d} x\right)\right]
\end{aligned}
$$

where $(\varphi+\varepsilon m)-v$ is the unit surplus inventory cost; $\int_{0}^{R q^{*}} F_{X}(x) \mathrm{d} x$ is the expected surplus inventory; $p-(\varphi+\varepsilon m)$ is the unit shortage cost; $\mu-\left(R q^{*}-\int_{0}^{R q^{*}} F_{X}(x) \mathrm{d} x\right)$ is expected quantity out of stock. We define

$$
C(q)=[(\varphi+\varepsilon m)-v] \int_{0}^{R q^{*}} F_{X}(x) \mathrm{d} x+[p-(\varphi+\varepsilon m)]\left[\mu-\left(R q^{*}-\int_{0}^{R q^{*}} F_{X}(x) \mathrm{d} x\right)\right]
$$

is the retailer's expected cost. Thus, $\frac{\partial \hat{\Pi}_{\alpha}^{*}}{\partial \alpha}>0$.

(c) We discuss the change of the overconfident retailer's optimal belief expected profit $\hat{\Pi}_{\alpha}^{*}$.

$$
\hat{\Pi}_{\alpha}^{*}-\Pi^{*}=(1-\alpha) \Pi^{*}+[p-(\varphi+\varepsilon m)] \alpha \mu-\Pi^{*}=\alpha\left[[p-(\varphi+\varepsilon m)] \mu-\Pi^{*}\right] .
$$

We know $[p-(\varphi+\varepsilon m)] \mu-\Pi^{*}=C\left(q^{*}\right)$ from the above proof. Thus, $\hat{\Pi}_{\alpha}^{*}-\Pi^{*}=\alpha C\left(q^{*}\right) \geq 0$. Moreover, $\hat{\Pi}_{\alpha}^{*}=\Pi^{*}$ if and only if $\alpha=0$.

Corollary 3.4 illustrates that the overconfident retailer's optimal belief expected profit is a function of the unbiased retailer's optimal expected profit $\Pi^{*}$ and increasing in overconfidence level. From the perspective of expected profit, this corollary clearly gives the reason why the overconfident retailer orders based on his own perceived demand distribution. The overconfident retailer considers that the profit from making a decision based on his beliefs is always greater than the profit he would have expected if he had been unbiased. Moreover, the excess profit is directly proportional to the level of overconfidence. To a greater extent, this encourages the behavior of overconfident retailer to make order decisions according to their own beliefs.

\section{EXTENDED OVERCONFIDENCE MODEL}

In Section 4, overconfident retailer simply has cognitive biases on the variance of demand. He overestimates his level of prediction, behaving as though demand is less variable than $X$. We extend to other cases, that is, overconfident retailer overestimates not only the level of forecasting, but also the level of marketing. He believes high demand for MC products. His thought of the mean demand is greater than the actual value. This kind of overconfident retailer is a retailer decide the order quantity $q_{o}$ as though the demand were $D_{o}$ rather than $X$, where

$$
D_{o}=\alpha k \mu+(1-\alpha) X
$$

for $\alpha \in[0,1]$ and $k \in\left[1, \infty^{+}\right)$.

We assume $D_{o}$ has a cumulative density function $(\mathrm{CDF}) F_{D_{o}}(x)$. We easily obtained $E\left[D_{o}\right]=(\alpha k+1-\alpha) \mu$, $\operatorname{Var}\left[D_{o}\right]=(1-\alpha)^{2} \sigma^{2}$. That is, the overconfident retailer's judgement of the mean and the variance of random market demand is at fault. It is easy to prove that the overconfident retailers' judgment of the mean of the market random demand is greater than the actual value, but their judgment of the variance of the market 


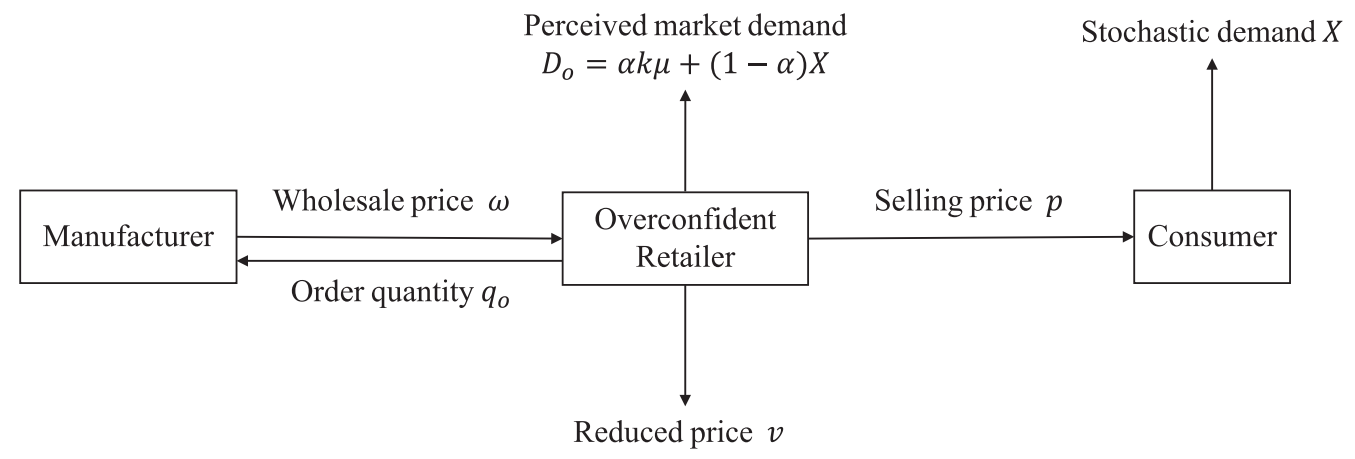

FiguRE 3. Extended overconfidence supply chain.

random demand is smaller than the actual value. We define the the parameter $\alpha$ and $k$ are respectively the level of overprecision and the level of overestimation (Fig. 3).

Then, the belief profit and belief expected profit function for the overconfident retailer, $\hat{\Pi}_{o}, E\left[\hat{\Pi}_{o}\right]$, can be expressed as

$$
\begin{aligned}
\hat{\Pi}_{o} & =p \min \left\{D_{o}, R q_{o}\right\}-(\varphi+\varepsilon m) R q_{o}+v\left(R q_{o}-D_{o}\right)^{+} \\
& =[p-(\varphi+\varepsilon m)] R q_{o}-(p-v)\left(R q_{o}-D_{o}\right)^{+} ; \\
E\left[\hat{\Pi}_{o}\right] & =[p-(\varphi+\varepsilon m)] R q_{o}-(p-v) \int_{o}^{R q_{o}} F_{D_{o}}(x) \mathrm{d} x .
\end{aligned}
$$

That's not how the profit actually incurred. The resulting expected profit for the retailer is

$$
E\left[\Pi_{o}\right]=[p-(\varphi+\varepsilon m)] R q_{o}-(p-v) \int_{o}^{R q_{o}} F_{X}(x) \mathrm{d} x
$$

when the retailer chooses the optimal order quantity $q_{o}^{*}$, we can express the optimal belief profit and resulting profit as follows:

$$
\begin{aligned}
& \hat{\Pi}_{o}^{*}=[p-(\varphi+\varepsilon m)] R q_{o}^{*}-(p-v) \int_{o}^{R q_{o}^{*}} F_{D_{o}}(x) \mathrm{d} x ; \\
& \Pi_{o}^{*}=[p-(\varphi+\varepsilon m)] R q_{o}^{*}-(p-v) \int_{o}^{R q_{o}^{*}} F_{X}(x) \mathrm{d} x .
\end{aligned}
$$

Theorem 4.1 characterizes the retailer's optimal order quantity $q_{o}^{*}$ in extended overconfidence model compared with the unbiased retailer's optimal order quantity $q^{*}$. The relationship with the modularity level $m$ is also described.

Theorem 4.1. Given the MC manufacturer's reliable level $R$,

(a) The overconfident retailer's optimal order quantity $q_{o}^{*}=R^{-1} \alpha k \mu+(1-\alpha) q^{*}$.

(b) The overconfident retailer's optimal order quantity $q_{o}^{*}$ is decreasing in the modularity level $m$.

Proof. (a) In the unbiased case, the retailer's optimal order quantity $q^{*}$ satisfies $F_{X}\left(R q^{*}\right)=\beta$ where $\beta$ is the critical fractile. In the extended model, the overconfident retailer with overconfidence level $\alpha$ and $k$ orders $q_{o}^{*}$ such that $F_{D_{o}}\left(R q_{o}^{*}\right)=\beta$ when demand is $X$. Thus, $F_{X}\left(R q^{*}\right)=F_{D_{o}}\left(R q_{o}^{*}\right)=\beta$. According to (3.16), $D_{o}=\alpha k \mu+(1-\alpha) X$. As a result,

$$
\begin{aligned}
F_{D_{o}}\left(R q_{o}^{*}\right) & =P\left(D_{o} \leq R q_{o}^{*}\right)=P\left(\alpha k \mu+(1-\alpha) X \leq R q_{o}^{*}\right) \\
& =P\left(X \leq \frac{R q_{o}^{*}-\alpha k \mu}{1-\alpha}\right)=F_{X}\left(\frac{R q_{o}^{*}-\alpha k \mu}{1-\alpha}\right) \\
& =\beta .
\end{aligned}
$$


Consequently, $R q^{*}=\frac{R q_{o}^{*}-\alpha k \mu}{1-\alpha}$, that is,

$$
q_{o}^{*}=R^{-1} \alpha k \mu+(1-\alpha) q^{*} .
$$

(b) According to Lemma 2.1, $\beta=\frac{p-(\varphi+\varepsilon m)}{p-v}$. Thus, we conclude that, in the extended model, the overconfident retailer's optimal order quantity $q_{o}^{*}$ is decreasing in the modularity level $m$.

Theorem 4.1 illustrates that the overconfident retailer's optimal order quantity $q_{o}^{*}$ in extended overconfidence model is a linear function of the unbiased retailer's optimal order quantity $q^{*}$. Moreover, if the modularity level of the MC manufacturer increases, the order quantity of the overconfident retailer decreases. Corollary 4.2 characterizes the relationship among $q^{*}, q_{\alpha}^{*}$ and $q_{o}^{*}$.

Corollary 4.2. Given the MC manufacturer's reliable level $R$,

(a) The overconfident retailer's optimal order quantity in extended overconfidence model $q_{o}^{*}$ is greater than that in the basic overconfidence model $q_{\alpha}^{*}$ in all cases, i.e., $q_{o}^{*}>q_{\alpha}^{*}$.

(b) The overconfident retailer's optimal order quantity in extended overconfidence model $q_{o}^{*}$ and that in the unbiased model $q^{*}$ satisfy $q_{o}^{*}<q^{*}$ when $q^{*}>R^{-1} k \mu ; q_{o}^{*}>q^{*}$ when $q^{*}<R^{-1} k \mu$ and $q_{o}^{*}=q^{*}=R^{-1} k \mu$ when $q^{*}=R^{-1} k \mu$.

Proof. (a) According to the proof of Theorems 3.1 and 4.1, $F_{D}\left(R q_{\alpha}^{*}\right)=F_{X}\left(\frac{R q_{\alpha}^{*}-\alpha \mu}{1-\alpha}\right)=\beta$ and $F_{D_{o}}\left(R q_{o}^{*}\right)=$ $F_{X}\left(\frac{R q_{o}^{*}-\alpha k \mu}{1-\alpha}\right)=\beta$. Thus, $\frac{R q_{\alpha}^{*}-\alpha \mu}{1-\alpha}=\frac{R q_{o}^{*}-\alpha k \mu}{1-\alpha}=\beta$. As a result,

$$
q_{o}^{*}-q_{\alpha}^{*}=R^{-1} \alpha \mu(k-1) .
$$

Because our assumption of $k>1, q_{o}^{*}-q_{\alpha}^{*}>0$ for all $\alpha$ and $k$.

(b) Because the overconfident retailer's optimal order quantity $q_{o}^{*}=R^{-1} \alpha k \mu+(1-\alpha) q^{*}$ in the extended model,

$$
q_{o}^{*}-q^{*}=R^{-1} \alpha k \mu-\alpha q^{*}=\alpha\left(R^{-1} k \mu-q^{*}\right) .
$$

If $q^{*}>R^{-1} k \mu$, then $q_{o}^{*}<q^{*}$. Similarly, $q_{o}^{*}>q^{*}$ when $q^{*}<R^{-1} k \mu$. If $q^{*}=R^{-1} k \mu, q_{o}^{*}=q^{*}=R^{-1} k \mu$.

Part (a) of Corollary 4.2 shows that the retailer's optimal order quantity in extended overconfidence model is always greater than that in the basic overconfidence model. This suggests, on the one hand, that the retailer in extended overconfidence model has a greater degree of overconfidence. Corollary $4.2 \mathrm{~b}$ reveals the conclusion as follow. The size comparison between $q^{*}$ and $R^{-1} k \mu$ determines the optimal order quantity relationship between the overconfident retailer in the extended model and the unbiased retailer. The definition of the market environment from Schweitzer and Cachon [25] is still quoted in the following Corollary 4.3.

Corollary 4.3. Given the MC manufacturer's reliable level $R$,

(a) The overconfident retailer's optimal order quantity $q_{o}^{*}$ and the level of overprecision $\alpha$ satisfy.

(i) Under a high-profit condition $\left(q^{*}>\mu\right)$, $\frac{\partial q_{o}^{*}}{\partial \alpha}<0$ when $q^{*}>R^{-1} k \mu$; $\frac{\partial q_{o}^{*}}{\partial \alpha}>0$ when $\mu<q^{*}<R^{-1} k \mu$.

(ii) Under a low-profit condition $\left(q^{*}<\mu\right), \frac{\partial q_{0}^{*}}{\partial \alpha}>0$ in all cases.

(b) The overconfident retailer's optimal order quantity $q_{o}^{*}$ and the level of overestimation $k$ satisfy $\frac{\partial q_{o}^{*}}{\partial k}>0$ in all cases. 
Proof. Prior analytical work has shown that the overconfident retailer's optimal order quantity is $q_{o}^{*}=R^{-1} \alpha k \mu+$ $(1-\alpha) q^{*}$ in the extended model. We take the derivative of $\alpha$ and $k$ respectively in $q_{o}^{*}$, then

$$
\begin{aligned}
& \frac{\partial q_{o}^{*}}{\partial \alpha}=R^{-1} k \mu-q^{*} \\
& \frac{\partial q_{o}^{*}}{\partial k}=R^{-1} \alpha \mu .
\end{aligned}
$$

Thus, $\frac{\partial q_{o}^{*}}{\partial \alpha}<0$ when $q^{*}>R^{-1} k \mu, \frac{\partial q_{o}^{*}}{\partial \alpha}>0$ when $\mu<q^{*}<R^{-1} k \mu$ and $\frac{\partial q_{o}^{*}}{\partial \alpha}>0$ when $q^{*}<\mu$. According to the definition of the market environment from Schweitzer and Cachon [25], the unbiased retailer's expected profit-maximizing order quantity is greater than mean demand for the case of high-profit, $q^{*}>\mu$, and less than mean demand for the case of low-profit, $q^{*}<\mu$. We combine these two analyses to come to Corollary 4.3a. Because $\frac{\partial q_{o}^{*}}{\partial k}=R^{-1} \alpha \mu>0$ is always true, Corollary $4.3 \mathrm{~b}$ is proved.

This result indicates how the overconfident retailer's optimal order quantity is affected by the level of overprecision and by the level of overestimation, respectively. The overconfident retailer's optimal order quantity in extended model is increasing in the overestimation level in all cases. However, the relationship between the overconfident retailer's optimal order quantity in extended model and overprecision level is more complex combining with the analysis of the level of market profit, which is shown in the Corollary 4.3a. The conclusion is similar to that in Corollary 3.2. The only difference is that the classification case, thus the description is omitted. We then present some conclusions about the retailer's optimal resulting expected profit and optimal belief expected profit in extended overconfidence model by the following theorem and corollary.

Theorem 4.4. Given the MC manufacturer's reliable level $R$,

(a) The overconfident retailer's optimal resulting expected profit in extended overconfidence model $\Pi_{o}^{*}$ is always less than the unbiased retailer's optimal expected profit $\Pi^{*} ;$ i.e., $\Pi_{o}^{*} \leq \Pi^{*}$;

(b) The overconfident retailer's optimal resulting expected profit in extended overconfidence model $\Pi_{o}^{*}$ and the level of overprecision $\alpha$ satisfy: $\frac{\partial \Pi_{o}^{*}}{\partial \alpha}<0$ and $\frac{\partial^{2} \Pi_{o}^{*}}{\partial \alpha^{2}}<0$;

(c) The overconfident retailer's optimal resulting expected profit in extended overconfidence model $\Pi_{o}^{*}$ and the level of overestimation $k$ satisfy: $\frac{\partial \Pi_{o}^{*}}{\partial k}<0$ when $q^{*}<R^{-1} k \mu, \frac{\partial \Pi_{o}^{*}}{\partial k}>0$ when $q^{*}>R^{-1} k \mu$ and $\frac{\partial^{2} \Pi_{o}^{*}}{\partial k^{2}} \leq 0$ in all cases.

Proof. (a) Same as the proof of Theorem 3.3, the expected profit for the unbiased retailer is $E[\Pi]=[p-(\varphi+$ $\varepsilon m)] R q-(p-v) \int_{0}^{R q} F_{X}(x) \mathrm{d} x$. Moreover, the first and second partial derivatives of the order quantity $q$ are $\frac{\partial E[\Pi]}{\partial q}=[p-(\varphi+\varepsilon m)] R-(p-v) R F_{X}(R q)$ and $\frac{\partial^{2} E[\Pi]}{\partial q^{2}}=-(p-v) R^{2} f(R q)$, respectively. Obviously, the retailer's expected profit function is concave in $q$ and maximized at $q^{*}$. Thus, whether the overconfident retailer's optimal order quantity $q_{o}^{*}$ in extended overconfidence model is higher or lower than the unbiased retailer's optimal order quantity $q^{*}$, it's always true that the overconfident retailer's resulting expected profit $\Pi_{o}^{*}$ in extended overconfidence model is less than the unbiased retailer's optimal expected profit $\Pi^{*}$.

(b) According to (4.4) and (4.6), the overconfident retailer's optimal resulting profit $\Pi_{o}^{*}$ in extended overconfidence model is

$$
\Pi_{o}^{*}=[p-(\varphi+\varepsilon m)] R\left[R^{-1} \alpha k \mu+(1-\alpha) q^{*}\right]-(p-v) \int_{o}^{R\left[R^{-1} \alpha k \mu+(1-\alpha) q^{*}\right]} F_{X}(x) \mathrm{d} x .
$$

We take the derivative of $\alpha$ to obtain

$$
\begin{aligned}
\frac{\partial \Pi_{o}^{*}}{\partial \alpha} & =[p-(\varphi+\varepsilon m)] R\left(R^{-1} k \mu-q^{*}\right)-(p-v) R\left(R^{-1} k \mu-q^{*}\right) F_{X}\left(R\left[R^{-1} \alpha k \mu+(1-\alpha) q^{*}\right]\right) \\
& =(p-v) R\left(R^{-1} k \mu-q^{*}\right)\left[\frac{p-(\varphi+\varepsilon m)}{p-v}-F_{X}\left[R\left(R^{-1} \alpha k \mu+(1-\alpha) q^{*}\right)\right]\right] \\
& =(p-v) R\left(R^{-1} k \mu-q^{*}\right)\left[F_{X}\left(R q^{*}\right)-F_{X}\left(R q_{o}^{*}\right)\right] .
\end{aligned}
$$


From the Corollary 4.2, $q_{o}^{*}<q^{*}$ when $q^{*}>R^{-1} k \mu$ and $q_{o}^{*}>q^{*}$ when $q^{*}<R^{-1} k \mu$. Similarly to the proof of Theorem 3.3, thus, $\frac{\partial \Pi_{o}^{*}}{\partial \alpha}<0$ is always true. Furthermore,

$$
\begin{aligned}
\frac{\partial^{2} \Pi_{o}^{*}}{\partial \alpha^{2}} & =-(p-v) R\left(R^{-1} k \mu-q^{*}\right)\left(k \mu-R q^{*}\right) f\left[R\left(R^{-1} \alpha k \mu+(1-\alpha) q^{*}\right)\right] \\
& =-(p-v)\left(k \mu-R q^{*}\right)^{2} f\left(R q_{o}^{*}\right)<0 .
\end{aligned}
$$

Accordingly, Theorem 4.4b is proved.

(c) We keep analyzing formula (4.10) in the above proof. We take the derivative of $k$ to obtain

$$
\begin{aligned}
\frac{\partial \Pi_{o}^{*}}{\partial k} & =[p-(\varphi+\varepsilon m)] \alpha \mu-(p-v) \alpha \mu F_{X}\left(R\left[R^{-1} \alpha k \mu+(1-\alpha) q^{*}\right]\right) \\
& =(p-v) \alpha \mu\left[\frac{p-(\varphi+\varepsilon m)}{p-v}-F_{X}\left(R q_{o}^{*}\right)\right] \\
& =(p-v) \alpha \mu\left[F_{X}\left(R q^{*}\right)-F_{X}\left(R q_{o}^{*}\right)\right] .
\end{aligned}
$$

In the same way, we use the conclusion in Corollary 4.2 , that is, $q_{o}^{*}<q^{*}$ when $q^{*}>R^{-1} k \mu$ and $q_{o}^{*}>q^{*}$ when $q^{*}<R^{-1} k \mu$. Also because $F_{X}(x)$ is an increasing function. We easily get $\frac{\partial \Pi_{o}^{*}}{\partial k}<0$ when $q^{*}<R^{-1} k \mu$, $\frac{\partial \Pi_{o}^{*}}{\partial k}>0$ when $q^{*}>R^{-1} k \mu$. Furthermore,

$$
\begin{aligned}
\frac{\partial^{2} \Pi_{o}^{*}}{\partial k^{2}} & =-(p-v) R\left(R^{-1} k \mu-q^{*}\right)\left(k \mu-R q^{*}\right) f\left[R\left(R^{-1} \alpha k \mu+(1-\alpha) q^{*}\right)\right] \\
& =-(p-v)\left(k \mu-R q^{*}\right)^{2} f\left(R q_{o}^{*}\right)<0 .
\end{aligned}
$$

Theorem $4.4 \mathrm{c}$ has been proved by the above calculations.

As with the basic overconfidence model, the retailer in extended overconfidence model still consistently has profit loss, compared to the unbiased retailer. The proof of Theorem 4.4a asserts that the overconfident retailer's optimal order quantity again deviates from the unbiased retailer's optimal order quantity. We can further see the relation between the retailer's optimal resulting expected profit of extended overconfidence model and the overestimated level and the overprecision level in Theorems $4.4 \mathrm{~b}$ and $4.4 \mathrm{c}$. The optimal resulting expected profit function is an increasing concave function about the overprecision level. The relation between the retailer's optimal resulting expected profit and the overestimated level depends on the comparison of $q^{*}$ and $R^{-1} k \mu$. However, that is not how profits occur in the perception of overconfident retailer. The analysis of his belief profit is shown in the following corollary.

Corollary 4.5. Given the MC manufacturer's reliable level $R$,

(a) The overconfident retailer's optimal belief expected profit in extended overconfidence model $\hat{\Pi}_{o}^{*}$ satisfies $\hat{\Pi}_{o}^{*}=(1-\alpha) \Pi^{*}+[p-(\varphi+\varepsilon m)] \alpha k \mu$;

(b) The overconfident retailer's optimal belief expected profit in extended overconfidence model $\hat{\Pi}_{\alpha}^{*}$ is increasing in $\alpha$; i.e., $\frac{\partial \hat{\Pi}_{o}^{*}}{\partial \alpha}>0$;

(c) The overconfident retailer's optimal belief expected profit in extended overconfidence model $\hat{\Pi}_{\alpha}^{*}$ is increasing in $k$; i.e., $\frac{\partial \hat{\Pi}_{o}^{*}}{\partial k}>0$;

(d) The overconfident retailer's optimal belief expected profit in extended overconfidence model $\hat{\Pi}_{o}^{*}$ is always greater than the unbiased retailer's optimal expected profit $\Pi^{*} ;$ i.e., $\hat{\Pi}_{o}^{*} \geq \Pi^{*}$.

Proof. (a) We know from the proof of Corollary 3.4 that the retailer in extended overconfidence model only takes into account his belief in random demand $D_{o}$ when calculating profit. The profit calculated in this way 
is the retailer's belief expected profit $E\left[\hat{\Pi}_{o}\right]$. Then, according to (4.4), the overconfident retailer's optimal

belief expected profit in extended overconfidence model is $\hat{\Pi}_{o}^{*}=[p-(\varphi+\varepsilon m)] R q_{o}^{*}-(p-v) \int_{o}^{R q_{o}^{*}} F_{D_{o}}(x) \mathrm{d} x$. We take the integral transformation of $x=\alpha k \mu+(1-\alpha) y$.

$$
\begin{aligned}
\hat{\Pi}_{o}^{*} & =[p-(\varphi+\varepsilon m)] R q_{o}^{*}-(p-v) \int_{0}^{R q^{*}} F_{D_{o}}[\alpha k \mu+(1-\alpha) y] \mathrm{d}(\alpha k \mu+(1-\alpha) y) \\
& =[p-(\varphi+\varepsilon m)] R q_{o}^{*}-(p-v)(1-\alpha) \int_{0}^{R q^{*}} F_{X}(y) \mathrm{d} y .
\end{aligned}
$$

Because $q_{o}^{*}=R^{-1} \alpha k \mu+(1-\alpha) q^{*}$ in Theorem 4.1,

$$
\begin{aligned}
\hat{\Pi}_{o}^{*} & =[p-(\varphi+\varepsilon m)] R\left[R^{-1} \alpha k \mu+(1-\alpha) q^{*}\right]-(p-v)(1-\alpha) \int_{0}^{R q^{*}} F_{X}(y) \mathrm{d} y \\
& =(1-\alpha)\left[(p-(\varphi+\varepsilon m)) R q^{*}-(p-v) \int_{0}^{R q^{*}} F_{X}(y) \mathrm{d} y\right]+[p-(\varphi+\varepsilon m)] \alpha k \mu \\
& =(1-\alpha) \Pi^{*}+[p-(\varphi+\varepsilon m)] \alpha k \mu .
\end{aligned}
$$

Thus, the relationship of $\hat{\Pi}_{o}^{*}$ and $\Pi^{*}$ in Corollary $4.5 \mathrm{a}$ is proved.

(b) We take the derivative of $\alpha$ in $\hat{\Pi}_{o}^{*}$.

$$
\frac{\partial \hat{\Pi}_{o}^{*}}{\partial \alpha}=[p-(\varphi+\varepsilon m)] k \mu-\Pi^{*}>[p-(\varphi+\varepsilon m)] \mu-\Pi^{*} .
$$

According to the proof of Corollary 3.4, $[p-(\varphi+\varepsilon m)] \mu-\Pi^{*}=C\left(q^{*}\right)>0$. Consequently, $\frac{\partial \hat{\Pi}_{o}^{*}}{\partial \alpha}>0$ is demonstrated.

(c) We keep analyzing the profit formula $\hat{\Pi}_{o}^{*}=(1-\alpha) \Pi^{*}+[p-(\varphi+\varepsilon m)] \alpha k \mu$. We take the derivative of $k$ in $\hat{\Pi}_{o}^{*}$.

$$
\frac{\partial \hat{\Pi}_{o}^{*}}{\partial k}=[p-(\varphi+\varepsilon m)] \alpha \mu>0 .
$$

(d) We discuss the change in the overconfident retailer's optimal belief expected profit in extended overconfidence model compared to the unbiased retailer's optimal profit.

$$
\begin{aligned}
\hat{\Pi}_{o}^{*}-\Pi^{*} & =(1-\alpha) \Pi^{*}+[p-(\varphi+\varepsilon m)] \alpha k \mu-\Pi^{*} \\
& =\alpha\left[(p-(\varphi+\varepsilon m)) k \mu-\Pi^{*}\right]>\alpha\left[(p-(\varphi+\varepsilon m)) \mu-\Pi^{*}\right] .
\end{aligned}
$$

Similarly, we know $[p-(\varphi+\varepsilon m)] \mu-\Pi^{*}=C\left(q^{*}\right)>0$. Thus, $\hat{\Pi}_{o}^{*}-\Pi^{*}>0$. Now that Corollary 4.5 is all proved.

The optimal belief expected profit in extended overconfidence model is a function of the unbiased retailer's optimal expected profit. Because the overconfident retailer of extended model overestimates the mean value of random market demand, similarly to Corollary 3.4, his optimal belief expected profit is always greater than the unbiased retailer's optimal profit. That is why the overconfident retailer makes a decision based on his beliefs. Moreover, the overconfident retailer's optimal belief expected profit of extended model is increasing in the overestimated level and the overprecision level, respectively. This result shows the more overconfident retailer is, the greater his belief profit is, leading him to be more willing to place orders according to their own will. 


\section{Conclusions}

In this paper, we combine the mass customization with a behavioral bias of overconfidence innovatively. We consider the supply chain which is made up of mass customization manufacturer and overconfident retailers and ultimate purchaser. Based on the newsvendor model, we study the retailer's optimal order-quantity decisions in the supply chain. The order quantity needs to be committed before the realization of random demand and the objective is to optimize profit. We first briefly discuss the conclusions of the newsvendor model in the case of unbiased retailers. Then two models are considered and theorems are proposed. We present the conclusions about the optimal order quantity and maximum profit in the two models. The first is to compare them with the basic model in which retailers are unbiased. Then these optimal order quantity and maximum profit are analyzed respectively with modularity level and overconfidence level. In particular, both performances of overconfidence level are discussed in the extended model. Compare the findings with the prior literature, some conclusions have been drawn. Firstly, this paper innovatively introduces overconfidence into the mass customization supply chain. Compared with the previous studies, this paper studies the influence of behavioral bias on the characteristic supply chain, and presents the relationship between the optimal decision and the modularity level of the manufacturer of mass customization products. Secondly, the yield rate of manufacturer of mass customization products introduced in this paper makes the conclusion of the optimal order quantity of overconfident retailers different from the conclusion of Croson et al. [3]. In other words, the existence of the yield rate increases the optimal order quantity of overconfident retailers.

By analyzing the above theoretical results, we provide the following managerial insights for decision makers of mass customization retailers and manufacturers.

From perspective of the retailers in MC supply chain, it's important for them to make clear that his behavioral bias of overconfidence could result the loss of profit. Moreover, the higher the overconfidence level is, the greater the profit loss is. In detail, the following three points are important for retailers. First, be sure to receive timely feedback to get more accurate market information. The retailers need to conduct regular investigations on market conditions. They should understand market dynamics as comprehensively as possible, such as consumer preferences and market trends. All feedback helps retailers make more accurate decisions. Second, it's necessary for retailers to break down the decision task. That is, break down the decision process into parts and specify the information needed to complete each part. This measure not only makes decisions more precise, but also reduces the potential impact of overconfidence. In addition to decision decomposition, analytic hierarchy process can be used. This requires the retailers to decompose the elements that are always relevant to the decision into levels of goals, guidelines, solutions, and so on. Third, imagine why your judgment might be wrong. For example, there may be behavioral biases, cognitive limitations, or information blocking. It means that the retailers should force themselves to consider information that does not confirm their beliefs. Taking these possible problems into full consideration and making a prudent decision will reduce the loss of the retailers.

For mass customization manufacturers, it is necessary to determine the appropriate modularity level. As shown in theoretical conclusions, the overconfident retailer's optimal order quantity is decreasing in the modularity level, whether in the basic overconfidence model or the extended model. However, if the modularity level is too low, it can be unable to meet the market demand, which will lead to the decline of its competitiveness as a mass customization enterprise. Therefore, the manufacturers should carefully determine the level of modularity in their company's production. On the one hand, manufacturers can survey the market demand and know consumers' preference for personalized products. On the other hand, manufacturers also need to consider the change of order quantity of downstream retailers. Both of these are important factors that affect the level of modularity.

From the supply chain's point of view, the repurchase contract mechanism can be considered to maximize the profit of the supply chain. It is of practical significance for manufacturers to use the repurchase contract mechanism to correct the irrational behavior of retailers and maximize the profit of the supply chain so as to maintain a long-term and stable relationship. The manufacturer may set a buyback value of the MC products that the retailer fails to sell, and the salvage value of the MC products is transferred to the manufacturer. 
There are many interesting problems that are worthwhile to study in future. First, overconfidence under other conditions is worth considering, such as the competitive retailers of MC supply chain. When both retailers are overconfident, it's harder to study if they have different overconfidence levels. Second, in this paper, the price of a certain mass customization product is fixed. The case that price varies with demand deserve consideration. In this case, how to determine the optimal order quantity is an interesting direction. Third, if the manufacturer of mass customization is the subject of research, then he should decide how to order from more upstream suppliers. This involves different order decisions at different modularity levels.

Acknowledgements. This work is partly supported by the National Natural Science Foundation of China under Grants 71671055, 72071056, 71971075, 71531008 and 71690230. This work is also partly supported by the National Key Research and Development Program of China 2019YFE0110300.

\section{REFERENCES}

[1] A. Alptekinoglu and C.J. Corbett, Mass customization vs. mass production: variety and price competition. Manuf. Serv. Oper. Manage. 10 (2008) 204-217.

[2] Z.M. Bi and W.J. Zhang, Modularity technology in manufacturing: taxonomy and issues. Int. J. Adv. Manuf. Technol. 18 (2001) 381-390.

[3] D. Croson, R, Croson and Y. Ren, How to Manage an Overconfident Newsvendor. Cox School of Business, Southern Methodist University, Dallas (2008).

[4] T. Choi, C. Ma, B. Shen and Q. Sun, Optimal pricing in mass customization supply chains with risk-averse agents and retail competition. Omega-Int. J. Manage. Sci. 88 (2019) 150-161.

[5] Q. Fei and L. Zhao, A competitive model of standardization and customization with behavior-based pricing. Proc. Comput. Sci. 159 (2019) 2374-2383.

[6] F.S. Fogliatto, G.J. Silveira and D. Borenstein, The mass customization decade: an updated review of the literature. Int. J. Prod. Econ. 138 (2012) 14-25.

[7] F. Gino and G. Pisano, Toward a theory of behavioral operations. Manuf. Serv. Oper. Manage. 10 (2008) 676-691.

[8] M.D. Grubb, Selling to overconfident consumers. Am. Econ. Rev. 99 (2009) 1770-1807.

[9] C. Gutierrez, T. Astebro and T. Obloj, The impact of overconfidence and ambiguity attitude on market entry. Org. Sci. 31 (2020) 308-329.

[10] B. Jiang and C. Liu, Managerial optimism in a competitive market. Prod. Oper. Manage. 28 (2019) $833-846$.

[11] T. Jain, J. Hazra and T.C. Cheng, Sourcing under overconfident buyer and suppliers. Int. J. Prod. Econ. 206 (2018) 93-109.

[12] P. Jost and T. Susser, Company-customer interaction in mass customization. Int. J. Prod. Econ. 220 (2020) 107454.

[13] S.N. Kirshner and L. Shao, The overconfident and optimistic price-setting newsvendor. Eur. J. Oper. Res. 277 (2019) $166-173$.

[14] A. Kocabiyikoglu, C.I. Gogus and M.S. Gonul, Decision making and the price setting newsvendor: experimental evidence. Decis. Sci. 47 (2016) 157-186.

[15] M. Li, Overconfident distribution channels. Prod. Oper. Manage. 28 (2019) 1347-1365.

[16] M. Li, N.C. Petruzzi and J. Zhang, Overconfident competing newsvendors. Manage. Sci. 63 (2017) $2637-2646$.

[17] B. MacCarthy, P.G. Brabazon and J. Bramham, Fundamental modes of operation for mass customization. Int. J. Prod. Econ. 85 (2003) 289-304.

[18] A. Merle, J. Chandon, E. Roux and F. Alizon, Perceived value of the mass customized product and mass customization experience for individual consumers. Prod. Oper. Manage. 19 (2010) 503-514.

[19] D. Moore and P. Healy, The trouble with overconfidence. Psychol. Rev. 115 (2008) 502-517.

[20] A. Ovchinnikov, B.B. Moritz and B.F. Quiroga, How to compete against a behavioral newsvendor. Prod. Oper. Manage. 24 (2015) 1783-1793.

[21] N.C. Petruzzi and M. Dada, Pricing and the newsvendor problem: a review with extensions. RAIRO: OR 47 (1999) 183-194.

[22] Y. Qi, Z. Mao, M. Zhang and H. Guo, Manufacturing practices and servitization: the role of mass customization and product innovation capabilities. Int. J. Prod. Econ. 228 (2020) 107747.

[23] Y. Ren and R. Croson, Overconfidence in newsvendor orders: an experimental study. Manage. Sci. 59 (2013) $2502-2517$.

[24] Y. Ren, D.C. Croson and R. Croson, The overconfident newsvendor. J. Oper. Res. Soc. 68 (2017) $496-506$.

[25] M.E. Schweitzer and G.P. Cachon, Decision bias in the newsvendor problem with a known demand distribution: experimental evidence. Manage. Sci. 46 (2000) 404-420.

[26] G.J. Silveira, D. Borenstein and F.S. Fogliatto, Mass customization: literature review and research directions. Int. J. Prod. Econ. 72 (2001) 1-13.

[27] D. Wu and F. Chen, The overconfident and ambiguity-averse newsvendor problem in perishable product decision. Comput. Ind. Eng. 148 (2020) 106689.

[28] Y. Xu, Y. Landon, S. Segonds and Y. Zhang, A decision support model in mass customization. Comput. Ind. Eng. 114 (2017) $11-21$. 
[29] L. Xu, X. Shi, P. Du, K. Govindan and Z. Zhang, Optimization on pricing and overconfidence problem in a duopolistic supply chain. Comput. Oper. Res. 101 (2019) 162-172.

[30] W. Xue, T. Choi and L. Ma, Diversification strategy with random yield suppliers for a mean-variance risk-sensitive manufacturer. Transp. Res. Part E-Logistics Transp. Rev. 90 (2016) 90-107.

[31] J. Yao and L. Liu, Optimization analysis of supply chain scheduling in mass customization. Int. J. Prod. Econ. 117 (2009) 197-211.

[32] L. Yao, Y.F. Chen and H. Yan, The newsvendor problem with pricing: extensions. Int. J. Manage. Sci. Eng. Manage. 1 (2006) $3-16$.

[33] X. Yong, M. Chu and W. Hongwei, A research on overconfident newsvendor model. Ind. Eng. J. 16 (2013) 38.

[34] M. Zhang, H. Guo, B. Huo, X. Zhao and J. Huang, Linking supply chain quality integration with mass customization and product modularity. Int. J. Prod. Econ. 207 (2017) 227-235. 